\title{
Role of paraspinal mapping before transforaminal epidural injections for lumbar radiculopathy
}

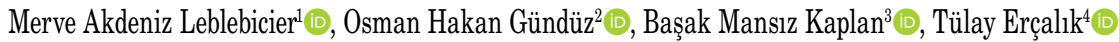 \\ ${ }^{1}$ Department of Physical Medicine and Rehabilitation, Health Sciences University, Evliya Çelebi Training and Research Hospital, Kütahya, Turkey \\ ${ }^{2}$ Department of Physical Medicine and Rehabilitation, Division of Pain Medicine, Marmara University School of Medicine, Istanbul, Turkey \\ ${ }^{3}$ Department of Physical Medicine and Rehabilitation, Health Sciences University, Ankara Training and Research Hospital, Ankara, Turkey \\ ${ }^{4}$ Department of Physical Medicine and Rehabilitation, Health Sciences University, Şişli Hamidiye Eftal Training and Research Hospital, Istanbul, Turkey
}

Received: July 10, 2019 Accepted: January 20, 2020 Published online: May 25, 2021

\begin{abstract}
Objectives: This study aims to investigate whether electromyography (EMG) including paraspinal mapping (PM) and specific clinical findings before the injection have a predictive role on the results in patients undergoing unilateral transforaminal epidural steroid injection (TFESI).

Patients and methods: In this prospective study, a total of 46 patients (19 males, 17 females; mean age: $44.0 \pm 10.8$ years; range, 23 to 60 years) with unilateral L5 root compression confirmed by physical examination, EMG and lumbar MRI between March 2013 and January 2014 were included. The patients underwent L5 TFESI. After the injection, they were examined at $1 \mathrm{~h}$, three weeks, and three months.

Results: The clinical findings and presence of acute involvement on EMG were not predictive for TFESI results; however, the patients with lower scores for the L5 segment in PM benefited more from the injection, compared to patients with higher scores for the L5 segment in PM. Conclusion: In patients with very clearly defined L5 radiculopathy, PM EMG can give us an idea about the effectiveness of L5 TFESI.

Keywords: Lumbar radiculopathy, paraspinal mapping, transforaminal epidural injection.
\end{abstract}

Low back pain represents a significant socio-economic problem, affecting individuals from all age groups. ${ }^{[1]}$ Epidural steroid injection (ESI) is an effective method of relieving symptoms in patients unresponsive to conservative treatment methods in lumbar radiculopathy. ${ }^{[2]}$

Although the studies have shown that ESI is effective in the short term, data about long-term outcomes are still contradictory. ${ }^{[3]}$ To date, many studies have investigated the factors affecting patients' response to ESI. Some data have been obtained on the predictive ability of clinical findings, imaging findings, and electromyography (EMG); however, clear conclusions have not been reached, yet. ${ }^{[4-6]}$
Electrophysiological examination is often used for diagnosis of lumbar radiculopathy. The needle EMG examination, including the paraspinal muscles, can detect lumbar root lesions with a high sensitivity. ${ }^{[7]}$ Previous studies have shown that transforaminal epidural steroid injection (TFESI) results are better in patients who have no neurogenic findings in needle EMG. ${ }^{[5]}$ These data indicate that the results are superior, as the diagnostic accuracy would be stronger in patients with EMG findings. ${ }^{[5]}$ The paraspinal mapping (PM) is obtained by scoring needle EMG findings of lumbar paraspinal muscles from four different points on the symptomatic side of the spine. The studies on PM are mostly focused on patients with spinal stenosis. ${ }^{[8,9]}$ There are only a few

Corresponding author: Merve Akdeniz Leblebicier, MD. SBÜ Evliya Çelebi Eğitim ve Araştırma Hastanesi Fizik Tedavi ve Rehabilitasyon Kliniği, 43040 Kütahya, Türkiye. e-mail: merve1985akdeniz@hotmail.com 
studies about its availability in lumbar radiculopathy that is not caused by stenosis.

In the present study, we hypothesized that paraspinal EMG score may be important for success in injection, although patients had single root compression in lumbar magnetic resonance imaging (MRI). We, therefore, aimed to investigate the predictive role of clinical findings and electrophysiologic examinations including PM before the injection in patients who had single-sided, single-root TFESI.

\section{PATIENTS AND METHODS}

This prospective study was conducted at Physical Medicine and Rehabilitation outpatient clinic of Marmara University, School of Medicine, Pendik Training and Research Hospital between March 2013 and January 2014. A total of 46 patients (19 males, 17 females; mean age: $44.0 \pm 10.8$ years; range, 23 to 60 years) with unilateral L5 root compression causing lower extremity radiculitis and/or radiculopathy as confirmed by physical examination, EMG and lumbar MRI were included. Clinical examination confirmed radiculopathy (signs of neurological dysfunction, including abnormalities of sensation, reflex, motor or gait, with associated nerve tension signs). ${ }^{[10]}$ Exclusion criteria were as follows: pregnancy and lactation, lumbar spinal stenosis, facet pain without radicular pain, spondylolisthesis-spondylolysis without radicular pain, cauda equina syndrome, progressive neurological deficit, presence of polyneuropathy, a history of previous lumbar surgery or ESI within the last six months or allergy to local anesthetics or corticosteroids. A written informed consent was obtained from each patient. The study protocol was approved by the Marmara University, School of Medicine, Ethics Committee (date/no: 09.2013.0062). The study was conducted in accordance with the principles of the Declaration of Helsinki.

Clinical examination, electrophysiological evaluation and ESI of the patients were made by three physicians who were unaware of one another, but not blinded to the patient's clinical complaint. In the clinical examination, lumbar range of motion (ROM), straight leg raising test (SLRT), muscle strength, and sensory examinations were made. Pain level, functional status, and mood were evaluated by Visual Analog Scale (VAS), Oswestry Disability Index (ODI), and Beck Depression Inventory (BDI), respectively.

\section{Measurements}

Nerve conduction studies (NCSs) on the lower extremity, needle EMG, and PM studies were performed in the electrophysiological evaluation. The studies were done using the Medtronic-Keypoint ${ }^{\bullet}$ device (Medtronic-Keypoint Denmark, 2007). The NCSs were performed with surface electrodes and included antidromic sensory conduction velocity (SCV), sensory nerve action potential (SNAP) amplitude, motor conduction velocity, motor or distal motor latency, compound muscle action potential (CMAP) amplitude, F-wave of the nerves served by the roots corresponding to the painful dermatome. The SNAP amplitude was measured from the first positive peak to the following negativity and that of CMAP from baseline to the following negative peak. Minimum F-wave (Fmin) latency elicited by 12 supramaximal stimuli at $1-\mathrm{Hz}$ without any facilitation technique was measured. Lower limb skin temperature was kept constant above $32^{\circ} \mathrm{C} .{ }^{[1]}$ During the NCS, F-wave latency of both tibial nerves was examined with tibial and common peroneal motor and sural sensory conduction studies on both lower extremities. In needle EMG, a $50-\mathrm{mm}$ monopolar EMG needle was used. Bilateral quadriceps, tibialis anterior, peroneus longus and the medial head of the gastrocnemius muscle were examined in needle EMG. Fibrillation potentials and positive sharp waves were used as the determinants of acute axonal degeneration. The number of phases, duration, amplitude, and recruitment was assessed. The chronic axonal degeneration of a root was defined with the presence of polyphasic, long duration and high amplitude motor unit action potentials with various degrees of decrease in recruitment. The limb EMG was categorized as normal, acute, or chronic radiculopathy. Superficial and deep paraspinal muscles in bilateral L2-5 segments were examined in a cranial-caudal direction in paraspinal EMG. In this procedure, patients were asked to lay in the prone position with a pillow under the abdomen. Superficial paraspinal muscles with mixed innervation and multifidus muscles with specific innervation were evaluated separately for each level. The PM was obtained as fibrillation potentials and positive sharp waves scored from 0 to 4 for each of 24 different points. Total PM score was summed by totaling all of the values. Also, in this study, different scoring was made for L5 myotome by the same method and included in the calculations as PM of L5 (L5 PM)..$^{[9,12]}$

\section{Epidural injection}

After the clinical and laboratory assessment, steroid and local anesthetic injection was performed 
transforaminally to the symptomatic side L5 nerve root using fluoroscopy guidance. When the injection site was identified on fluoroscopy, the skin and subcutaneous tissue anesthesia were provided with a short-acting local anesthetic agent. A 22-gauge spinal needle was placed into the epidural space by using intermittent fluoroscopic imaging. After confirming the position of the needle tip under the pedicle in the lateral view, 0.5 to $1 \mathrm{~mL}$ of contrast was injected to confirm epidural distribution and to rule out any intravascular injection. Then, a mixture of $80 \mathrm{mg}$ methylprednisolone acetate, $1 \mathrm{~mL} 0.5 \%$ bupivacaine, and $1 \mathrm{~mL}$ saline was injected.

\section{Data analysis}

All patients were observed for $1 \mathrm{~h}$ after injection and re-examined. The VAS scores for pain at $1 \mathrm{~h}$ were recorded. The patients were re-examined at three weeks and three months. Functional and mood status of the patients were evaluated. The patients with a decrease of $\geq 80 \%$ in the control VAS scores were compared with those having $<80 \%$ in the control VAS scores regarding the clinical and electrophysiological features.

\section{Statistical analysis}

Statistical analysis was performed using the IBM SPSS version 22.0 software (IBM Corp., Armonk, NY, USA). Descriptive data were expressed in mean \pm standard deviation (SD), median (min-max) or number and frequency. The appropriateness of the parameters to a normal distribution was evaluated using the Shapiro-Wilks test. The Student's t-test was used to compare normally distributed quantitative variables between the two groups, while the Mann-Whitney $U$ test was used to compare non-normally distributed variables between the two groups. The Fisher's exact test, chi-square test, and Yates's correction for continuity were used for the comparison of qualitative data. The Spearman's rank correlation test was used to examine the relationship between the parameters. A $p$ value of $<0.05$ was considered statistically significant.

\section{RESULTS}

Of all patients included in the study, 36 completed three-month follow-up period. Ten patients were unable to be medically examined due to the following reasons: one had a second injection after three weeks, one was lost to follow-up, and the remaining eight had lumbar disc surgery within at an average of 2.3 months after the epidural injection. These eight patients were also analyzed as the surgery group in this study. Demographic and clinical characteristics of the patients are given in Table 1.

A total of 36 patients, including 19 females and 17 males, were evaluated at three weeks and three months. The VAS scores, functional status, and mood changes all significantly improved after the treatment ( $\mathrm{p}=0.001$ and $\mathrm{p}<0.01$, respectively).

The patients were divided into two subgroups according to the decline in VAS scores $(\geq 80 \%$, and $<80 \%)$ and all patients achieved a decline at three weeks and three months (Table 2). Before injection,

\begin{tabular}{|c|c|c|c|c|c|c|}
\hline \multicolumn{7}{|c|}{$\begin{array}{l}\text { TABLE } 1 \\
\text { Demographic and clinical characteristics of study population }\end{array}$} \\
\hline & \multicolumn{3}{|c|}{ Treatment group $(\mathrm{n}=36)$} & \multirow[b]{2}{*}{ Min-Max } & \multicolumn{2}{|c|}{ Surgery group $(n=9)$} \\
\hline & $\mathrm{n}$ & $\%$ & Mean \pm SD & & $\mathrm{n}$ & Min-Max \\
\hline Age (year) & & & $43.9 \pm 10.8$ & $23-60$ & & $26-55$ \\
\hline Height $(\mathrm{cm})$ & & & $165.3 \pm 8.7$ & $150-183$ & & $160-180$ \\
\hline Weight (kg) & & & $74.0 \pm 11.5$ & $55-100$ & & $60-95$ \\
\hline Body mass index $\left(\mathrm{kg} / \mathrm{m}^{2}\right)$ & & & $27 \pm 3.5$ & $21.5-36.7$ & & $23.4-31.2$ \\
\hline Duration of symptoms (month) & & & $3.9 \pm 2.4$ & $1-12$ & & $1-6$ \\
\hline \multicolumn{7}{|l|}{ Sex } \\
\hline Female & 19 & 52.8 & & & 3 & \\
\hline Male & 17 & 47.2 & & & 5 & \\
\hline \multicolumn{7}{|l|}{ Side of root compression } \\
\hline Right & 20 & 55.6 & & & 4 & \\
\hline Left & 16 & 44.4 & & & 4 & \\
\hline
\end{tabular}




\begin{tabular}{|c|c|c|c|c|c|}
\hline \multicolumn{6}{|c|}{$\begin{array}{c}\text { TABLE } 2 \\
\text { The relationship between improvement of VAS scores and variables before injection }\end{array}$} \\
\hline & \multicolumn{4}{|c|}{ Improvement of VAS at the $3^{\text {rd }}$ week } & \multirow[b]{3}{*}{$p$} \\
\hline & \multicolumn{2}{|c|}{$\geq 80 \%$} & \multicolumn{2}{|r|}{$<80 \%$} & \\
\hline & $\%$ & Mean \pm SD & $\%$ & Mean \pm SD & \\
\hline Oswestry Disability Index score before injection & & $33.4 \pm 10.6$ & & $32.7 \pm 6.0$ & $0.804^{1}$ \\
\hline Beck Depression Inventory score before injection & & $12.9 \pm 7.9$ & & $14.3 \pm 9.3$ & $0.647^{1}$ \\
\hline VAS before injection & 6 & $6.8 \pm 2.1$ & 8 & $8.2 \pm 1.7$ & $0.022^{\star 2}$ \\
\hline VAS difference before injection and at $1^{\text {st }} \mathrm{h}(\%)$ & 100 & $93 \pm 12.4$ & 80 & $68.7 \pm 30.9$ & $0.006^{\star 2}$ \\
\hline PM score & 5 & $7.1 \pm 6.0$ & 8 & $8.5 \pm 6.4$ & $0.006^{\star 2}$ \\
\hline \multirow[t]{4}{*}{ L5 PM score } & 2 & $3 \pm 3.2$ & 2 & $2.5 \pm 2.7$ & $0.712^{2}$ \\
\hline & \multicolumn{4}{|c|}{ Improvement of VAS at $3^{\text {rd }}$ month } & \\
\hline & \multicolumn{2}{|c|}{$\geq 80 \%$} & \multicolumn{2}{|c|}{$<80 \%$} & \\
\hline & $\%$ & Mean \pm SD & $\%$ & Mean \pm SD & $p$ \\
\hline Oswestry Disability Index score before injection & & $32.6 \pm 9.5$ & & $33.2 \pm 6.4$ & $0.830^{1}$ \\
\hline Beck Depression Inventory score before injection & & $15.6 \pm 8.9$ & & $12.3 \pm 8.5$ & $0.271^{1}$ \\
\hline VAS before injection & 7.5 & $7.6 \pm 2.4$ & 7 & $7.8 \pm 1.5$ & $0.612^{2}$ \\
\hline VAS difference before injection and at $1^{\text {st }} \mathrm{h}(\%)$ & 92.8 & $86.1 \pm 16.9$ & 80 & $70.5 \pm 33.4$ & $0.191^{2}$ \\
\hline PM score & 5.5 & $8.1 \pm 6.7$ & 6.5 & $8.0 \pm 5.9$ & $0.848^{2}$ \\
\hline L5 PM score & 1 & $1.4 \pm 1.9$ & 3 & $3.7 \pm 3.1$ & $0.020^{\star 2}$ \\
\hline
\end{tabular}

the clinical examination, functional status (ODI) and mood (BDI) scores were not significantly different between the groups. In the second group whose VAS decline was $\geq 80 \%$ at three weeks, pre-injection VAS scores were significantly lower, and the VAS recovery was greater than the first group at $1 \mathrm{~h}$. There was no significant difference between the groups at three months $(\mathrm{p}<0.05)$.

The presence of abnormal spontaneous activity on L5 paraspinal mapping score (PMS) had no significant effect on the results. The PMS was similar in both groups. The L5 paraspinal score for the patients who had a three-month VAS decline of $\geq 80 \%$ was significantly lower. Also, there was a negative relationship between the three-month VAS decline and L5 PMS ( $r=-0.67)$. The relationship between the improvement of VAS scores and variables before injection are given in Table 2 and Figure 1.

To determine the characteristics of patients who responded to TFESI negatively and later underwent surgery, the patients were analyzed in two subgroups. These patients were compared regarding clinical and electrophysiological characteristics before the injection. There were no significant differences regarding age and body mass index values between these two groups
( $>0.05)$. The differences between treatment and surgery groups are given in Table 3 . The SLRT before TFESI was significantly different in patients who underwent surgery, compared to those who were not operated $(\mathrm{p}<0.01)$. The SLRT, in patients undergoing

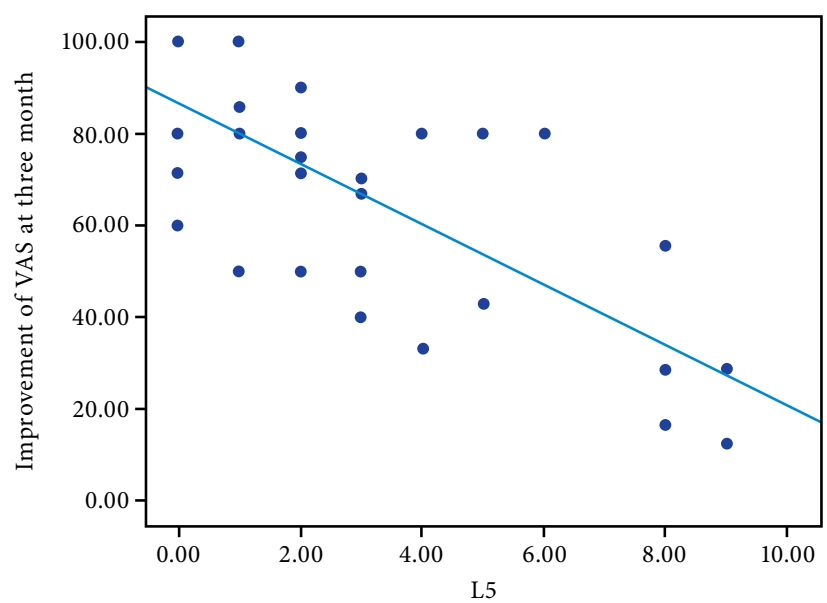

Figure 1. There is a negative linear relationship between improvement of VAS score at three months (vertical axis) and L5 PM (horizontal axis). As the L5 PM increases, there is a tendency for a lower VAS improvement to TFESI at three months.

VAS: Visual Analog Scale; PM: Paraspinal mapping; TFESI: Transforaminal epidural steroid injection. 


\begin{tabular}{|c|c|c|c|c|c|c|c|}
\hline \multicolumn{8}{|c|}{$\begin{array}{c}\text { TABLE } 3 \\
\begin{array}{c}\text { Difference between treatment and surgery groups. The SLRT before TFESI was significantly different in patients who went to } \\
\text { surgery, compared to those who were not operated }(\mathrm{p}<0.001)\end{array}\end{array}$} \\
\hline & \multicolumn{3}{|c|}{ Treatment group $(n=36)$} & \multicolumn{3}{|c|}{ Surgery group $(\mathrm{n}=8)$} & \multirow[b]{2}{*}{$p$} \\
\hline & $\mathrm{n}$ & $\%$ & Mean \pm SD & $\mathrm{n}$ & $\%$ & Mean \pm SD & \\
\hline SLRT before injection & & & & & & & $0.001^{\star \star 2}$ \\
\hline $20^{\circ}-30^{\circ}$ & 0 & 0 & & 3 & 37.5 & & \\
\hline $30^{\circ}-40^{\circ}$ & 0 & 0 & & 5 & 62.5 & & \\
\hline $40^{\circ}-50^{\circ}$ & 9 & 25 & & 0 & & & \\
\hline $50^{\circ}-60^{\circ}$ & 3 & 8.3 & & 0 & & & \\
\hline $60^{\circ}-70^{\circ}$ & 8 & 22.2 & & 0 & & & \\
\hline Negative & 16 & 44.4 & & 0 & & & \\
\hline VAS difference before injection and after injection & & 84 & $78.0 \pm 26.4$ & & 45 & $44.6 \pm 5.8$ & $0.001^{\star * 1}$ \\
\hline PM score & & 6 & $8 \pm 6.1$ & & 17 & $16.3 \pm 5.6$ & $0.004^{* 1}$ \\
\hline L5 PM score & & 2 & $2.7 \pm 2.9$ & & 7 & $7 \pm 1.1$ & $0.001^{\star * 1}$ \\
\hline Beck Depression Inventory score before injection & & 12.5 & $13.8 \pm 8.7$ & & 21.5 & $21.1 \pm 9.2$ & $0.028^{\star 1}$ \\
\hline Oswestry Disability Index score before injection & & 32.5 & $32.9 \pm 7.8$ & & 44 & $44.5 \pm 4.4$ & $0.001^{\star * 1}$ \\
\hline
\end{tabular}

surgery, ranged from $30^{\circ}$ to $40^{\circ}$. The lowest SLRT positivity starting value in the treatment group was observed at $40^{\circ}$. The SLRT was found to be negative in $44.4 \%$ cases. The injection decrease in pain was lower in the operated patients, compared to the treatment group $(\mathrm{p}<0.001)$. The PMS measurements and L5 levels were significantly higher in patients undergoing surgery, followed by the group $(\mathrm{p}<0.01)$. The PMS and L5 paraspinal EMG score in patients undergoing surgery were significantly higher, compared to the treatment group $(\mathrm{p}<0.01)$. Pre-treatment BDI and ODI scores in patients undergoing surgery were significantly higher than the treatment group $(\mathrm{p}<0.05$ and $\mathrm{p}<0.01$, respectively).

\section{DISCUSSION}

In this study, we demonstrated that, in a population with clearly defined single level L5 radiculopathy caused by disc herniation, clinical findings and presence of abnormal spontaneous activity on EMG were not predictive for the TFESI results. However, patients with lower scores for L5 segment in the PM benefited more from the TFESI, compared to patients with higher scores for L5 segment in the PM. Moreover, we found that pain reduction within $1 \mathrm{~h}$ after the TFESI was predictive for short-term results, although it was not determinant in the long-term.

In our study, we used $\geq 80 \%$ reduction in the VAS scores as the success rate, consistent with previous studies in the literature. ${ }^{[13]}$ In a study which examined the effective factors in pain reduction after TFESI, McCormick et al. ${ }^{[14]}$ evaluated 188 patients retrospectively and, similar to our results, clinical examination findings before the injection, functional status, mood features were not found to be determinants for the outcomes. The superiority of our study is that it was prospective and included a more specific group of patients, that is, with the involvement of a single spinal nerve root (L5). We found that short-term results were worse in the patients with high baseline VAS scores. This finding is inconsistent with the study of McCormick et al. ${ }^{[14]}$ This can be attributed to the use of different study designs, including a patient group with more specific properties, rather than a large group of patients, and the difference in the study method.

In the literature, there is a limited number of studies regarding the predictive role of the electrophysiological findings. In a study of Fish et al., ${ }^{[15]}$ they found that the presence of acute or chronic involvement on EMG had no effect in pain reduction after TFESI in 39 patients. In this study, the patients had injection at multiple levels. Similarly, Marchetti et al. ${ }^{[16]}$ found no significant difference in their study including 89 patients. Annaswamy et al. $^{[17]}$ reported that the results were better in the presence of acute involvement, but several different lumbar levels and interlaminar epidural injection were used in this study. In another retrospective study including 170 patients, the results were found 
to be better in patients with EMG findings, regardless of differentiating acute and chronic, and this finding was attributed to the diagnostic value of EMG. ${ }^{[5]}$ In our study, all patients had acute or chronic EMG findings and the presence of acute involvement did not affect the success rate. Although the findings of our study are compatible with the other studies, it was studied prospectively in a more specific patient group with a more specific transforaminal technique. Moreover, PMS and its L5 PM EMG scores which were not previously studied in this patient group were evaluated in this study. As expected, there was no significant finding in the total PMS. Thus, patients with lumbar spinal stenosis or multilevel lumbar disc herniations were excluded, and only patients with L5 radiculopathy were included. The L5 PM EMG scores before the injection were significantly lower in the patients with $\geq 80 \%$ improvement at three months, compared to the other patients. Until now, studies on the PMS are concentrated in patients diagnosed with spinal stenosis. There is no study, except for the diagnostic value in patients with lumbar radiculopathy. ${ }^{[18,19]}$ Our study is, therefore, the first in the literature and is valuable with its guiding feature for future studies.

It is also the most precise study addressing the question of whether the burden of denervation predicts outcome from injection. While all previous studies address a more general question of whether EMG can localize the root in question and, thus, improve injection, none of them address the more precise pathophysiological question posed in this study. Among individuals who have very certain lesions at one particular root, the severity of denervation can predict the impact of an ESI.

In the present study, eight patients undergoing surgery within an average of 2.3 months after the injection and 36 patients at three months of follow-up were also compared, and a significant difference between pre-treatment measurements for SLRT. This finding indicates that SLRT is more significant in determining the prognosis before the injection, but only SLRT may be inadequate for the decision to be referred for surgery. However, the surgical treatment can be primarily considered, rather than re-injection in the follow-up of patients with SLRT below $40^{\circ}$. We also found that the VAS improvement at $1 \mathrm{~h}$ is inadequate in patients undergoing surgery.

Furthermore, abnormal spontaneous activity was positive on L5 paraspinal EMG in all of the patients undergoing surgery, while this rate was found to be $69.4 \%$ in the other patients. The L5 PM EMG scores were significantly higher in the group undergoing surgery. In this case, instead of a diagnostic evaluation made with the examination of only the lower extremity muscles by needle EMG before treatment, an examination in the related paraspinal muscles according to the affected nerve root is recommended. It may help to predict the results before TFESI with the PMS for the related segment. Although paraspinal EMG score is significant in patients with single-level root involvement, this situation may be different in patients with multiple-level root involvement. Despite MRI findings, paraspinal scores including the affected levels may prove helpful for nerve root level needed to treat. We believe that there is a need for methodological researches about the possible relationship needle EMG and epidural injection.

The lack of a control group is the main limitation of the present study. Further comprehensive, largescale, prospective, randomized-controlled studies are needed to confirm these findings.

In conclusion, among individuals with very clearly defined single level radiculopathy caused by disc herniation, clinical status and PMS are of utmost importance in tailoring the treatment. Also, The SLRT is critical in identifying the prognosis before the injection. The VAS at $1 \mathrm{~h}$ after the procedure can be helpful in predicting long-term outcomes. In our study, the L5 PM EMG scores were higher in the operated patients and our study is the first to show the response was worse in patients with high paraspinal EMG scores for the related level, compared to VAS. Although paraspinal EMG is painful process, it may be advisable to perform it, as it is essential to make a prognosis in advance of performing an ESI.

\section{Declaration of conflicting interests}

The authors declared no conflicts of interest with respect to the authorship and/or publication of this article.

Funding

The authors received no financial support for the research and/or authorship of this article.

\section{REFERENCES}

1. Gelalis ID, Arnaoutoglou E, Pakos EE, Politis AN, Rapti $\mathrm{M}$, Xenakis TA, et al. Effect of interlaminar epidural steroid injection in acute and subacute pain due to lumbar disk herniation: a randomized comparison of 2 different protocols. Open Orthop J 2009;3:121-4.

2. Manchikanti L, Pampati V, Falco FJ, Hirsch JA. An updated assessment of utilization of interventional pain management techniques in the Medicare population: 2000 - 2013. Pain Physician 2015;18:E115-27. 
3. Manchikanti L, Knezevic NN, Boswell MV, Kaye AD, Hirsch JA. Epidural Injections for Lumbar Radiculopathy and Spinal Stenosis: A Comparative Systematic Review and Meta-Analysis. Pain Physician 2016;19:E365-410.

4. Ghahreman A, Bogduk N. Predictors of a favorable response to transforaminal injection of steroids in patients with lumbar radicular pain due to disc herniation. Pain Med 2011;12:871-9.

5. McCormick Z, Cushman D, Caldwell M, Marshall B, Ghannad L, Eng C, et al. Does Electrodiagnostic Confirmation of Radiculopathy Predict Pain Reduction after Transforaminal Epidural Steroid Injection? A Multicenter Study. J Nat Sci 2015;1:e140.

6. Sivaganesan A, Chotai S, Parker SL, Asher AL, McGirt MJ, Devin CJ. Predictors of the efficacy of epidural steroid injections for structural lumbar degenerative pathology. Spine J 2016;16:928-34.

7. Dillingham TR, Lauder TD, Andary M, Kumar S, Pezzin LE, Stephens RT, et al. Identifying lumbosacral radiculopathies: an optimal electromyographic screen. Am J Phys Med Rehabil 2000;79:496-503.

8. Ozcan-Eksi EE, Yagci I, Erkal H, Demir-Deviren S. Paraspinal muscle denervation and balance impairment in lumbar spinal stenosis. Muscle Nerve 2016;53:422-30.

9. Haig AJ, London Z, Sandella DE. Symmetry of paraspinal muscle denervation in clinical lumbar spinal stenosis: support for a hypothesis of posterior primary ramus stretching? Muscle Nerve 2013;48:198-203.

10. Manson NA, McKeon MD, Abraham EP. Transforaminal epidural steroid injections prevent the need for surgery in patients with sciatica secondary to lumbar disc herniation: a retrospective case series. Can J Surg 2013;56:89-96.

11. Wilbourn AJ, Aminoff MJ. AAEM minimonograph 32: the electrodiagnostic examination in patients with radiculopathies. American Association of Electrodiagnostic Medicine. Muscle Nerve 1998;21:1612-31.

12. Lee JH, Lee SH. Physical examination, magnetic resonance image, and electrodiagnostic study in patients with lumbosacral disc herniation or spinal stenosis. J Rehabil Med 2012;44:845-50.

13. Sencan S, Ozcan-Eksi EE, Cil H, Tay B, Berven S, Burch S, et al. The effect of transforaminal epidural steroid injections in patients with spondylolisthesis. J Back Musculoskelet Rehabil 2017;30:841-6.

14. McCormick Z, Cushman D, Casey E, Garvan C, Kennedy DJ, Plastaras C. Factors associated with pain reduction after transforaminal epidural steroid injection for lumbosacral radicular pain. Arch Phys Med Rehabil 2014;95:2350-6.

15. Fish DE, Shirazi EP, Pham Q. The use of electromyography to predict functional outcome following transforaminal epidural spinal injections for lumbar radiculopathy. J Pain 2008;9:64-70.

16. Marchetti J, Verma-Kurvari S, Patel N, Ohnmeiss DD. Are electrodiagnostic study findings related to a patient's response to epidural steroid injection? PM R 2010;2:1016-20.

17. Annaswamy TM, Bierner SM, Chouteau W, Elliott AC. Needle electromyography predicts outcome after lumbar epidural steroid injection. Muscle Nerve 2012;45:346-55.

18. Tong HC. Incremental ability of needle electromyography to detect radiculopathy in patients with radiating low back pain using different diagnostic criteria. Arch Phys Med Rehabil 2012;93:990-2.

19. Forero JJ, Ortiz-Corredor F, Díaz-Ruiz J, Lozano-Castillo A, Mendoza-Pulido C. Changes in electromyographic results of patients with lumbar radiculopathy: a follow-up study. Arch Phys Med Rehabil 2013;94:1287-92. 